\title{
Precise predictions of the mass of the discovered Higgs boson in supersymmetric scenarios
}

\author{
Henning Bahl, ${ }^{a}$ Nick Murphy ${ }^{b}$ and Heidi Rzehak ${ }^{c, *}$ \\ ${ }^{a}$ Deutsches Elektronen-Synchrotron DESY, \\ Notkestraße 85, 22607 Hamburg, Germany \\ ${ }^{b}$ CP3-Origins, University of Southern Denmark, \\ Campusvej 55, 5230 Odense M, Denmark ${ }^{\dagger}$ \\ ${ }^{c}$ Institute for Theoretical Physics, University of Tübingen, \\ Auf der Morgenstelle 14, 72076 Tübingen, Germany \\ E-mail: henning.bahl@desy.de, nickodel4@gmail.com, \\ heidi.rzehak@itp.uni-tuebingen.de
}

The mass of the discovered Higgs boson is one of its most precisely measured properties with an experimental accuracy at the sub-percent level. Besides its coupling behaviour, which conforms with the prediction of the Standard Model so far, the measured Higgs mass value can place strong constraints on extensions of the Standard Model, in particular supersymmetric ones. To fully exploit this experimental accuracy, a very precise prediction of the mass of the Standard Model-like Higgs boson in the respective model is required. In these proceedings, we comment on different methods to calculate the mass and present some recent developments in the effort of improving the predictions within the Minimal Supersymmetric Standard Model. In particular, we consider scenarios with heavy supersymmetric partner particles but relatively light Higgs bosons with and without CP-violation.

\footnotetext{
*** The European Physical Society Conference on High Energy Physics (EPS-HEP2021), *** *** 26-30 July 2021 ***

*** Online conference, jointly organized by Universität Hamburg and the research center DESY ***
}

\footnotetext{
* Speaker

${ }^{\dagger}$ Former academic affiliation
} 


\section{Introduction}

One of the best measured properties of the discovered Higgs boson is its mass with a value of $m_{H}=125.09 \pm 0.21$ (stat) \pm 0.11 (syst) $\mathrm{GeV}$ according to the combined Run 1 results of ATLAS and CMS [1]. Hence, the experimental accuracy reaches already the order of $O(100 \mathrm{MeV})$. In extensions of the Standard Model (SM), in particular in the Minimal Supersymmetric Standard Model (MSSM), the SM-like Higgs-boson mass can be predicted from other model parameters ${ }^{1}$. Quantum corrections can shift its value beyond the upper tree-level mass bound given by the $\mathrm{Z}$ boson mass. Therefore, the value of the mass of the discovered Higgs-boson constrains the viable parameter space significantly. In order to fully exploit the remarkably accurate measurement, the theoretical uncertainty originating from missing higher-order corrections due to the truncation of the perturbation theory needs to be at least at the same level as the experimental accuracy. The theoretical uncertainty depends on the specific scenario but is in general not much below $O(1 \mathrm{GeV})$, see discussions in Refs. [3, 4]. In addition, quantum corrections also change the mixing of the Higgs bosons leading to CP-odd admixtures in scenarios with CP-violation in the Higgs sector.

\section{The fixed-order approach}

In general, the Higgs-boson-mass spectrum in the MSSM is obtained by evaluating the determinant of the two-point vertex function $\hat{\Gamma}$,

$$
\hat{\Gamma}_{h H A}\left(p^{2}\right)=\mathrm{i}\left[p^{2} 1-\operatorname{diag}\left(m_{h}^{2}, m_{H}^{2}, m_{A}^{2}\right)+\hat{\Sigma}\left(p^{2}\right)\right]
$$

where, in this formula, the contribution of the Goldstone bosons is neglected. The zeros of the determinant correspond to the poles of the inverse of the two-point vertex function and hence the squared poles masses of the considered Higgs boson. The masses $m_{h}^{2}, m_{H}^{2}, m_{A}^{2}$ are the tree-level masses of the light and heavy CP-even and of the CP-odd Higgs boson, respectively, and $\hat{\Sigma}$ the renormalized self energy. In a fixed-order approach the self energies are evaluated up to a fixed order in perturbation theory, see for example the reviews [4, 5].

In the expression for the self energies, logarithms of mass ratios appear, such as the logarithm of the ratio of the mass of the top squarks $m_{\tilde{t}}$ and of the top quark $m_{t}$. These logarithms can become large if the one mass is much heavier than the other, requiring higher-order corrections to be included possibly beyond the feasible fixed-order calculation. In such a case, a different approach, the effective field theory (EFT) approach, is more appropriate.

\section{The EFT approach}

In the EFT approach, first a hierarchy of masses and a corresponding tower of EFTs is identified. Following Ref. [6], we consider the MSSM with heavy supersymmetric partner particles and relatively light Higgs bosons. At the scale of the soft-breaking mass parameter $M_{\mathrm{SUSY}}$, the full MSSM is matched to a type III Two-Higgs-Doublet Model (2HDM) without an additional $Z_{2}$ symmetry and both Higgs doublets coupling to top and bottom quarks in such a way that, at $M_{\mathrm{SUSY}}$,

\footnotetext{
${ }^{1}$ In the MSSM, the lightest Higgs-boson is in general considered to be the Standard-Model-like Higgs boson (see for example Ref. [2] for a benchmark scenario where this is not the case).
} 


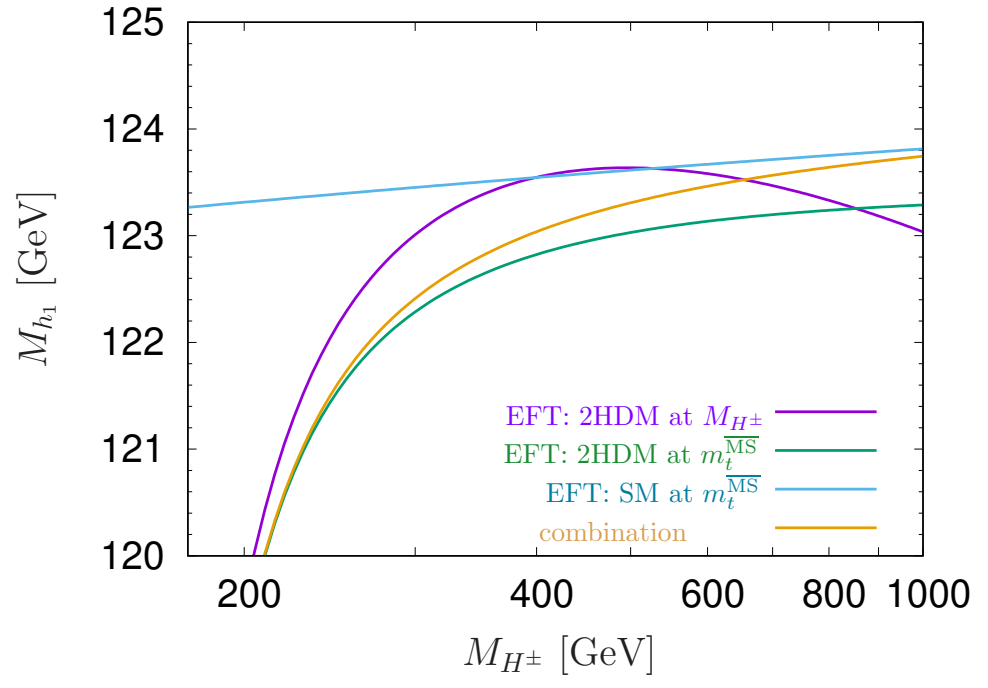

Figure 1: The mass of the SMlike Higgs boson $M_{h_{1}}$ in dependence of the mass of the charged Higgs boson $M_{H}^{ \pm}$. The different colours depict the different towers of EFT applied (see text for details). The SUSY soft breaking mass parameter is $M_{\mathrm{SUSY}}=$ $3 \mathrm{TeV}$, the absolute values of the trilinear couplings $A_{t}, A_{b}$ as well as the Higgs mixing parameter $\mu$ are $\left|A_{t}\right|=\left|A_{b}\right|=|\mu|=3 M_{\text {SUSY }}$ and $\tan \beta=5$. The phases are set to zero.

the MSSM and the 2HDM describe the same physics. Then, the 2HDM parameters are evolved down to a low scale applying two-loop renormalization group equations derived using Refs. [7] and compared to Refs. [8]. The mass of the SM-like Higgs boson $M_{h_{1}}$ evaluated at the low scale is shown in dependence of the mass of the charged Higgs boson $M_{H}^{ \pm}$in Fig. 1 for four different treatments at the low scale:

1. The calculation of the Higgs mass spectrum is performed at the scale $M_{H}^{ \pm}$within the 2HDM (violet line in Fig. 1).

2. The Higgs mass spectrum is calculated at the scale of the $\overline{\mathrm{MS}}$ top-quark mass $m_{t}^{\overline{\mathrm{MS}}}$ within the 2HDM (green line in Fig. 1).

3. At the scale $M_{H}^{ \pm}$, the $2 \mathrm{HDM}$ is matched to the SM. The resulting SM couplings are evolved down to the scale $m_{t}^{\overline{\mathrm{MS}}}$, at which the Higgs-boson mass is evaluated (blue line in Fig. 1).

4. In this approach we combine the first two and the third case: A tree-level matching to the SM is performed at the scale $M_{H^{ \pm}}$and the SM-quartic coupling $\lambda^{\mathrm{SM}}$, the SM-top-Yukawa coupling $y_{t}$, the strong coupling constant $g_{s}$ as well as the vacuum expectation value are evolved down to the scale $m_{t}$. Then, the $\mathcal{M}_{22}$ element of the Higgs mass matrix in the $\left(\phi_{1}, \phi_{2}, a_{1}, a_{2}\right)$ basis with the CP-even and the CP-odd components $\phi_{1}, \phi_{2}, a_{1}, a_{2}$, respectively, is replaced by $\mathcal{M}_{22} \rightarrow \mathcal{M}_{22}+\gamma^{\text {resum }} / \sin ^{2} \beta$ where $\gamma^{\text {resum }}=v^{2}\left(m_{t}\right) \lambda\left(m_{t}\right)-v^{2}\left(M_{H^{ \pm}}\right) \lambda\left(M_{\boldsymbol{H}^{ \pm}}\right)$, see also Ref. $[9,10]$ for a similar approach. (yellow line in Fig. 1).

In the calculation we allowed for non-vanishing phases in order to be sensitive for possible CPviolating effects. The matching of the MSSM to the 2HDM was performed at one-loop order, i.e.

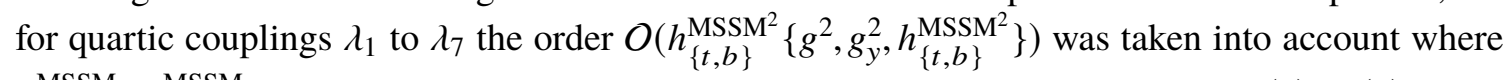
$h_{t}^{\mathrm{MSSM}}\left(h_{b}^{\mathrm{MSSM}}\right.$ ) are the top (bottom) MSSM Yukawa couplings and $g, g_{y}$ the $S U(2), U(1)$ gauge couplings, respectively $[10,11]$. For the Yukawa couplings in the 2HDM $h_{t}, h_{b}, h_{t}^{\prime}, h_{b}^{\prime}$, one-loop contribution of the order $O\left(h_{\{t, b\}}^{\mathrm{MSSM}}\left\{h_{\{t, b\}}^{\mathrm{MSSM}^{2}}, g_{s}^{2}\right\}\right)$ are included. The primed Yukawa couplings are the 'wrong' Yukawa couplings that only receive a non-zero value due to the one-loop threshold 
contributions. The Yukawa couplings of the first and second generation are set to zero and ignored. The calculation of the mass spectrum at the low scale within the 2HDM includes one-loop Yukawa contributions but neglects all higher-order corrections beyond these ones.

As can be seen in Fig. 1, the result of variant 4 (yellow) where also logs of the form $\log \left(M_{H}^{ \pm} / m_{t}\right)$ are resummed interpolates between the results of the 2HDM EFT for small $M_{H}^{ \pm}$(violet/green) and of the SM EFT at $m_{t}$ for large $M_{H}^{ \pm}$(blue). Increasing the value of $\tan \beta$ leads to higher values of the Higgs-boson mass and a faster approach of the interpolating result to the SM EFT.

\section{Combining fixed-order approach and EFT method: The hybrid approach}

The EFT approach is particularly useful if large mass differences appear, since it resums large logarithms up to all orders. It is required for a precise prediction, if very heavy particles occur. The fixed-order approach has the advantage that it is complete up to the considered order including non-logarithmic terms. Also, moderate mass differences are automatically taken into account.

In order to make use of the advantages of both, the fixed-order and the EFT approach, hybrid approaches combining these methods have been considered, see e.g. Refs. [12].

For the combination we have applied the following two steps: First, the Higgs fields of fixedorder calculation are redefined to match the normalization of the Higgs fields in the EFT [10, 13]. Second, the individual results of EFT and fixed-order calculation are added such that no double counting of logarithms appears. Therefore, the self energies in Eq. (1) need to be replaced by

$$
\hat{\Sigma}_{i j}^{\text {hybrid }}\left(p^{2}\right)=\hat{\Sigma}_{i j}^{\text {fixed order }}\left(p^{2}\right)+\Delta_{i j}^{\mathrm{EFT}}-\Delta_{i j}^{\mathrm{sub}}
$$

where $\hat{\Sigma}_{i j}^{\mathrm{fixed} \text { order }}\left(p^{2}\right)$ is the fixed-order self energy, $\Delta_{i j}^{\mathrm{EFT}}$ the EFT contribution to the mass matrix and $\Delta_{i j}^{\text {sub }}$ the subtraction terms to avoid the double counting of logarithms [10]. This procedure allows

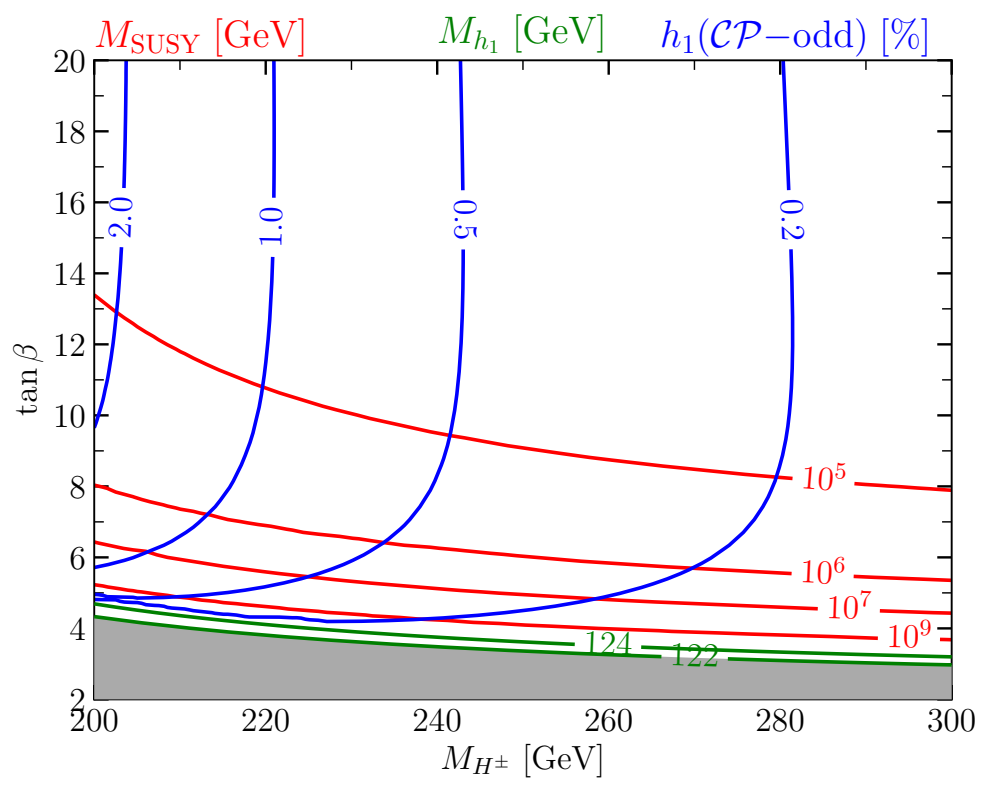

Figure 2: The CP-odd admixture to the SM-like Higgs boson (blue), the mass of the SM-like Higgs boson $M_{h_{1}}$ (green) and the values of $M_{\text {SUSY }}$ (red) are shown in the $\tan \beta-M_{H}^{ \pm}$plane. The values of $M_{\text {SUSY }}$ are chosen in such a way that in most of the plane $M_{h_{1}}=125 \pm 3 \mathrm{GeV}$. The values of the absolute values of the trilinear couplings and the Higgs mixing parameter are $\left|A_{t}\right|=\left|A_{b}\right|=|\mu|=3 M_{S}$ and the corresponding phases $\varphi_{A}=$ $2 \pi / 3, \varphi_{M_{3}}=\pi / 4$ where $\varphi_{M_{3}}$ is the phase of the gluino soft breaking mass parameter. The figure is taken from Ref. [14]. 
to calculate not only the mass but also the mixing of the Higgs bosons. As described in Ref. [14], we have combined the EFT calculation of Sect. 3 with the fixed-order calculation implemented in FeynHiggs [15], however, in the EFT part, we neglected the bottom Yukawa contributions in contrast to Sect. 3 but improved the accuracy of the threshold contributions and the extraction of the pole masses with respect to the previous calculation: For the threshold contributions for the 2HDM quartic couplings $\lambda_{1}$ to $\lambda_{7}$, also purely electroweak contributions of one-loop order as well as $O\left(\alpha_{t} \alpha_{s}\right)$ contributions following the approach described in Ref. [9] and using the results of [16] have been taken into account. Additionally, electroweak one-loop contributions to the thresholds of the 2HDM Yukawa couplings have been added. Furthermore, the matching between the SM and the 2HDM is performed at one-loop level instead of tree level only and the extraction of the mass of SM-like Higgs boson includes full one- and two-loop-order contributions [17].

In Fig. 2, the CP-odd admixture to the SM-like Higgs boson is shown in the $\tan \beta-M_{H}^{ \pm}$plane as blue contour lines. The green lines depict the mass of the SM-like Higgs boson and the grey region is the area where its mass is below $122 \mathrm{GeV}$ and the red lines show the value of $M_{\mathrm{SUSY}}$ that is needed to reach a Higgs mass of about $125 \mathrm{GeV}$. It is obvious that the CP-odd-admixture decreases strongly with increasing mass of the charged Higgs boson. It should also be noted that the charged Higgs boson is assumed to be very light with values only up to $300 \mathrm{GeV}$, and that these low values of $M_{H \pm}$ are strongly disfavoured by in particular B-physics observables. This shows clearly that the CP-odd admixture is already strongly constrained by both the mass value of the SM-like Higgs boson as well as of the charged Higgs boson without considering further constraints such as those from the electric dipole measurements. While the CP-violating effects are tiny for the SM-like Higgs boson in the MSSM, the heavy Higgs bosons can still have both, a CP-even and a CP-odd component.

\section{Conclusion}

We discussed different methods to calculate the Higgs-boson mass spectrum and their advantages. In the case of large mass differences, an EFT approach is required to resum large logarithms while the fixed-order approach takes small mass differences automatically into account. A combination of both, the hybrid approach, can be advantageous. We extended existing calculations to allow for CP-violating effects: The CP-odd admixture to the SM-like Higgs boson is tiny in the MSSM, most likely immeasurable at the LHC, however, CP-violating effects can appear between the heavy Higgs bosons in the MSSM.

\section{Acknowledgements}

H.B. acknowledges support by the Deutsche Forschungsgemeinschaft (DFG, German Research Foundation) under Germany's Excellence Strategy - EXC 2121 "Quantum Universe"-390833306. H.R.'s and N.M.'s work was partially funded by the Danish National Research Foundation, grant number DNRF90. H.R. was also partly supported by the German Federal Ministry for Education and Research (BMBF) under contract no. 05H18VFCA1 and partly by the Deutsche Forschungsgemeinschaft (DFG, German Research Foundation)_project no. 442089526. Part of this work was supported by a STSM Grant from COST Action CA16201 PARTICLEFACE. 


\section{References}

[1] ATLAS, CMS collaboration, G. Aad et al., Phys. Rev. Lett. 114 (2015) 191803.

[2] E. Bagnaschi et al., Eur. Phys. J. C 79 (2019) 617.

[3] H. Bahl, S. Heinemeyer, W. Hollik, G. Weiglein, Eur. Phys. J. C 80 (2020) no.6, 497.

[4] P. Slavich et al., Eur. Phys. J. C 81 (2021) no.5, 450.

[5] P. Draper, H. Rzehak, Phys. Rept. 619, 1 (2016).

[6] N. Murphy, H. Rzehak, arXiv: 1909.00726 [hep-ph].

[7] M. Machacek, M. Vaughn, Nuclear Physics B 222 (1983) 83; Nuclear Physics B 236 (1984) 221; Nuclear Physics B 249 (1985) 70; M.-x. Luo, H.-w. Wang, Y. Xiao, Phys. Rev. D67 (2003) 065019; I. Schienbein, F. Staub, T. Steudtner, K. Svirina, Nucl. Phys. B 939 (2019) 1; M. Sperling, D. Stöckinger, A. Voigt, JHEP 01 (2014) 068; JHEP 07 (2013) 132.

[8] J. Oredsson, J. Rathsman, JHEP 02 (2019) 152; A. E. Thomsen, Eur. Phys. J. C 81 (2021) no.5, 408 .

[9] G. Lee, C. E. M. Wagner, Phys. Rev. D92 (2015) 075032.

[10] H. Bahl, W. Hollik, JHEP 07 (2018) 182.

[11] M. Carena, J. Ellis, J. S. Lee, A. Pilaftsis, C. E. M. Wagner, JHEP 02 (2016) 123; H. E. Haber, R. Hempfling, Phys. Rev. D48 (1993) 4280; M. Gorbahn, S. Jäger, U. Nierste, S. Trine, Phys. Rev. D 84 (2011) 034030.

[12] T. Hahn, S. Heinemeyer, W. Hollik, H. Rzehak, G. Weiglein, Phys. Rev. Lett. 112 (2014) 141801; H. Bahl, W. Hollik, Eur. Phys. J. C76 (2016) 499; P. Athron, J. Park, T. Steudtner, D. Stöckinger, A. Voigt, JHEP 01 (2017) 079; F. Staub, W. Porod, Eur. Phys. J. C77 (2017) 338; H. Bahl, S. Heinemeyer, W. Hollik, G. Weiglein, Eur. Phys. J. C78 (2018) 57; P. Athron et al., Comput. Phys. Commun. 230 (2018) 145.

[13] H. Bahl, JHEP 02 (2019) 121.

[14] H. Bahl, N. Murphy, H. Rzehak, Eur. Phys. J. C 81, no.2, 128 (2021).

[15] S. Heinemeyer, W. Hollik, G. Weiglein, Comput. Phys. Commun. 124 (2000) 76; Eur. Phys. J. C9 (1999) 343; G. Degrassi, P. Slavich, F. Zwirner, Nucl. Phys. B611 (2001) 403; A. Brignole, G. Degrassi, P. Slavich, F. Zwirner, Nucl. Phys. B631 (2002) 195; Nucl. Phys. B643 (2002) 79; G. Degrassi, S. Heinemeyer, W. Hollik, P. Slavich, G. Weiglein, Eur. Phys. J. C28 (2003) 133; A. Dedes, G. Degrassi, P. Slavich, Nucl. Phys. B672 (2003) 144; S. Heinemeyer, W. Hollik, H. Rzehak, G. Weiglein, Eur. Phys. J. C39 (2005) 465; M. Frank, T. Hahn, S. Heinemeyer, W. Hollik, H. Rzehak, G. Weiglein, JHEP 02 (2007) 047; S. Heinemeyer, W. Hollik, H. Rzehak, G. Weiglein, Phys. Lett. B652 (2007) 300-309; T. Hahn, S. Heinemeyer, W. Hollik, H. Rzehak, G. Weiglein, Comput. Phys. Commun. 180 (2009) 1426; W. Hollik, S. Paßehr, Phys. Lett. B733 (2014) 144; JHEP 10 (2014) 171, Eur. Phys. J. C75 (2015) 336; T. Hahn, S. Paßehr, Comput. Phys. Commun. 214 (2017) 91.

[16] H. Bahl, I. Sobolev, G. Weiglein, Eur. Phys. J. C 80, no.11, 1063 (2020).

[17] D. Buttazzo et al., JHEP 12 (2013) 089. 